\title{
Medical Management of COVID-19: Evidence and Experience
}

\author{
Subhasish Bose ${ }^{a, j}$, Sreedhar Adapa ${ }^{\mathrm{b}}$, Narothama Reddy Aeddula ${ }^{\mathrm{c}}$, Sasmit Roy ${ }^{\mathrm{a}}$, \\ Deepak Nandikanti $^{\mathrm{d}}$, Phani Mohan Vupadhyayula ${ }^{\mathrm{e}}$, Srikanth Naramala ${ }^{\mathrm{f}}$, \\ Vijay Gayam, Vijayadershan Muppidi ${ }^{\text {h, Venu Madhav Konala }}{ }^{\mathrm{i}}$
}

\begin{abstract}
Coronavirus disease 2019 is caused by severe acute respiratory syndrome coronavirus 2 (SARS-CoV-2), and this infectious disease is termed COVID-19 in short. On a global scale, as of June 1, 2020, the World Health Organization (WHO) published statistics of $6,057,853$ infected patients and 371,166 deaths worldwide. Despite reported observational data about the experimental use of certain drugs, there is no conclusively proven curative therapy for COVID-19 as of now; however, remdesivir received emergency use authorization (EUA) by the Food and Drug Administration (FDA) recently for use in patients hospitalized with COVID-19. There are several ongoing clinical trials related to the pharmacological choices of therapy for COVID-19 patients; however, drug trials related to observational studies so far have yielded mixed results and therefore have created a sense of confusion among healthcare professionals (HCPs). In this review article, we seek to collate and provide a summary of treatment strategies for COVID-19 patients with a variable degree of illness and discuss pharmacologic and other therapies intended to be used either as experimental medi-
\end{abstract}

Manuscript submitted May 4, 2020, accepted May 20, 2020

Published online June 4, 2020

aDivision of Nephrology, Department of Internal Medicine, University of Virginia, Lynchburg, VA 24501-1167, USA

bDivision of Nephrology, Department of Internal Medicine, Adventist Medical Center, Hanford, CA 93230, USA

'Division of Nephrology, Department of Internal Medicine, Deaconess Health System Inc, Evansville, IN 47710, USA

${ }^{\mathrm{d} K i d n e y ~ S p e c i a l i s t s ~ o f ~ S o u t h e r n ~ N e v a d a ~(K S O S N), ~ L a s ~ V e g a s, ~ N V ~ 89030, ~}$ USA

${ }^{\mathrm{e} C a r o m o n t ~ R e g i o n a l ~ M e d i c a l ~ C e n t e r, ~ G a s t o n i a, ~ N C ~ 28054, ~ U S A ~}$

fDivision of Rheumatology, Department of Internal Medicine, Adventist Medical Center, Hanford, CA 93230, USA

gDepartment of Medicine, Interfaith Medical Center, Brooklyn, NY 11213, USA

hDepartment of Internal Medicine, IU Health Ball Memorial Hospital, Muncie, IN 47303, USA

iDivison of Medical Oncology, Department of Internal Medicine, Ashland Bellefonte Cancer Center, Ashland, KY 41169, USA

${ }^{j}$ Corresponding Author: Subhasish Bose, Division of Nephrology, Department of Internal Medicine, University of Virginia, Lynchburg, VA 24501-1167, USA.Email: drsbose@gmail.com

doi: https://doi.org/10.14740/jocmr4201 cine/therapy or as part of supportive care in complicated cases of COVID-19.

Keywords: Coronavirus disease 2019; SARS-CoV-2; Pharmacological treatment; Medical management; HIV; Transplant; ESKD

\section{Introduction}

Coronavirus disease 2019 is caused by severe acute respiratory syndrome coronavirus 2 (SARS-CoV-2), and this infectious disease is termed COVID-19 in short. The disease was first officially reported in December 2019 in Wuhan, China, and has spread globally since then, leading to the current COVID-19 pandemic. As of June 1, 2020, the Centers for Disease Control and Prevention (CDC) reported that a total of $1,787,680$ people were COVID-19 positive in the USA, and there were totally 104,396 individuals lost their lives to COVID-19 so far [1]. On a global scale, as of the same date, the World Health Organization (WHO) published statistics of $6,057,853$ infected patients and 371,166 deaths [2].

The symptoms of infected people with this novel coronavirus can range from being asymptomatic or minimal respiratory symptoms to febrile illness, as well as severe respiratory failure needing ventilator support. Despite reported observational data about the experimental use of certain drugs, there is no proven curative therapy for COVID-19 as of now; however, remdesivir received emergency use authorization (EUA) by the Food and Drug Administration (FDA) recently for use in patients hospitalized with COVID-19. Most interventions and guidelines for the management of the COVID-19 pandemic are related to prevention, isolation, and supportive treatment strategies. There are several ongoing clinical trials related to the pharmacological choices of therapy for COVID-19 patients; however, drug trials related to observational studies so far have yielded mixed results and therefore have created a sense of confusion among healthcare professionals (HCPs). In this review article, we seek to collate and provide a summary of treatment strategies for COVID-19 patients with a variable degree of illness, and discuss pharmacologic and other therapies intended to be used either as experimental medicine/therapy or as part of supportive care in complicated cases of COVID-19. 


\section{General Issues Around Medical Management of COVID-19}

\section{Use of non-steroidal anti-inflammatory drug (NSAID)}

Some anecdotal reports suggested association of NSAID use early during COVID-19 and progression to severe disease in young adults [3]. However, there has been no populationbased research showing the direct relationship of NSAID use and severe COVID-19. WHO and the United States National Institutes of Health (NIH) COVID-19 Treatment Guidelines Panel do not recommend NSAIDs being avoided when clinically indicated [4]. Acetaminophen should be used as the preferred antipyretic agent for fever associated with COVID-19; NSAID, if used, should be administered in the lowest effective dose; this approach is consistent with an established general approach to fever reduction in adults. It is also not recommended to discontinue NSAIDs in patients who are on them chronically for other conditions, unless there are clinical rationale to stop them (e.g., acute renal injury, gastrointestinal (GI) bleeding).

\section{Role of glucocorticoids}

In the past, glucocorticoids have been associated with an increased risk for mortality in patients with influenza and delayed viral clearance in patients with Middle East respiratory syndrome coronavirus (MERS-CoV) infection. Although they were widely used in the management of severe acute respiratory syndrome (SARS), there was no good evidence for benefit, and there was persuasive evidence of adverse short- and long-term harm [5]. Therefore, $\mathrm{CDC}$ recommended against the use of systemic glucocorticoids in patients with COVID-19, unless there are other indications such as asthma or chronic obstructive lung disease exacerbation, refractory septic shock, and adrenal insufficiency. However, their administration in critically ill patients with COVID-19-related adult respiratory distress syndrome (ARDS) is controversial. Based on data suggesting the potential benefit of glucocorticoids in patients with all-cause ARDS, the Society of Critical Care Medicine (SCCM) provided a conditional, weak recommendation in favor of glucocorticoids in patients with COVID-19 who have severe ARDS. If clinicians choose to administer glucocorticoids, the SCCM suggests that they should begin within the first 14 days, doses should be low, and courses should be short (e.g., intravenous (IV) dexamethasone $20 \mathrm{mg}$ once daily for 5 days, then $10 \mathrm{mg}$ once daily for 5 days). Data from retrospective Chinese cohort [6] regarding this topic were not without flaws, and hence large-scale prospective data will be required to settle this dilemma.

\section{Venous thromboembolism prophylaxis}

Patients with COVID-19 may carry an increased risk for systemic thrombosis. This is suggested by published case reports of pulmonary embolism in patients with COVID-19, and several cohorts reported to have elevated D-dimer levels and other markers of dysregulated coagulation [7]. Moreover, many patients hospitalized with COVID-19 have a higher risk of thromboembolism anyways because of advanced age and existing comorbidities. Additionally, markers of dysregulated coagulation and evidence of disseminated intravascular coagulation (DIC) have been seen to be associated with more severe disease and death in patients with COVID-19 [7]. However, the impact of anticoagulation (prophylactic or therapeutic) on the outcome of COVID-19 remains unknown. As with all hospitalized patients, pharmacologic prophylaxis of venous thromboembolism is recommended even in COVID-19 patients, typically using low-molecular-weight heparin, unless there are clinical contraindications such as active bleeding or severe thrombocytopenia.

\section{Use of nebulized medications}

Inhaled medications should be administered by metered-dose inhaler and not through a nebulizer. This is to avoid the risk of aerosolization and transmission of SARS-CoV-2. If nebulized therapy must be used, patients should be in an airborne infection isolation room (preferably negative pressure room), and any healthcare worker who needs to be in that room should use the contact and airborne precautions with appropriate personal protection equipment (PPE) including N95 mask with goggles and face shield or equivalent. It is also recommended not to re-enter the room for $2-3 \mathrm{~h}$ following nebulizer treatment for risk of aerosolized transmission of the virus.

\section{Empiric use of antibiotics for superimposed bacterial pneumonia}

Clinical features of COVID-19 are challenging to distinguish from bacterial pneumonia. Empiric treatment for bacterial pneumonia may also be reasonable in patients with documented COVID-19 if there is clinical suspicion. Empiric antibiotic therapy, if initiated, should be guided by microbial diagnosis (e.g., sputum Gram stain and culture) and reevaluate the need to continue antibiotic therapy daily. A low procalcitonin level usually is helpful to suggest against bacterial pneumonia; however, elevated procalcitonin has been described in the late stage of COVID-19 and does not necessarily indicate bacterial pneumonia [8].

\section{Continuation of Chronic Medical Therapy: Some Special Circumstances}

\author{
Angiotensin-converting enzyme inhibitors (ACEIs) or \\ angiotensin receptor blockers (ARBs)
}

It was suspected that COVID-19 patients who are receiving these agents might be at increased risk for adverse outcomes, 
but there is no clinical evidence that supports such speculation. Conversely, ARBs were proposed to have potential protective effects based on their mechanism of action [9], but there is no conclusive evidence to support this hypothesis. Multiple experts and guidelines recommended that patients who are already on ACEI or ARB should continue treatment with these agents if there is no other reason for discontinuation (e.g., hypotension, acute kidney injury (AKI)) [10].

\section{Statins}

Concern has been raised regarding potential hepatotoxicity from statins in COVID-19 patients who commonly demonstrate elevated transaminase levels. However, most evidence indicates that liver injury from statins is uncommon. On the other hand, statins are known inhibitors of the myeloid differentiation primary response 88 (MYD88) pathway, which results in marked inflammation, and have been reported to stabilize MYD88 levels in the setting of external stress in vitro and animal studies. Dysregulation of MYD88 has been noted and associated with poor outcomes in SARS-CoV and MERS$\mathrm{CoV}$ infections, but this has not been described with SARSCoV-2 [11].

\section{Immunomodulatory agents}

Initiation of immunosuppressive agents has been associated with increased risk for severe disease with respiratory viruses. However, no evidence routinely discontinuing treatment is of any benefit. Therefore, the plan to discontinue prednisone, biologics, or other immunosuppressive drugs in the setting of COVID-19 must be determined on a case-by-case basis. COVID-19-negative patients with underlying conditions requiring treatment with these agents should not be taken off suddenly, as discontinuing of these medications may result in loss of response when the agent is reintroduced. Statements from different medical societies support the approach of continuing immunomodulatory therapy in patients without infection [12].

\section{Symptomatic management}

Fever is the most common symptom and was noted in $88.7 \%$ of the COVID-19 patients in a study in China [13]. Acetaminophen is the recommended antipyretic in COVID-19. It can also be used in headache and myalgias. NSAIDs could be used, at the lowest effective dose, as an alternative antipyretic or pain reliever despite some report of NSAID use and worsening of COVID-19 severity [3]. Aspirin causes Reye syndrome, and should be avoided in children. Cough (often dry cough) is another prominent and common symptom of COVID-19. Cough medications may contain antihistamines and decongestants, and so must be used with caution. It is recommended that metered-dose inhalers are used, and nebulizers are avoided as much as possible due to the increased risk of aerosolization and the spread of the virus [14]. Hypotension and shock are potential complications of COVID-19. Vasopressors are pre- ferred to aggressive fluid resuscitation in patients with shock to avoid volume overload due to concern for the development of ARDS in these patients [15]. The use of empiric antibiotics and glucocorticoid therapy is controversial and has been discussed earlier in this article.

\section{Management of respiratory failure}

Viral infection causes inflammatory cytokine release and thereby edema in various vascular beds, usually subpleural in the early stages and alveolar edema in later stages [16]. Vascular endothelial damage in COVID-19 disrupts pulmonary vascular autoregulation in response to hypoxia and contributes to ventilation-perfusion (VQ) mismatch [17]. Moreover, inflammation of the alveolar lining, as well as decreased fluid clearance, leads to alveolar collapse and edema. The respiratory mechanics, pathology, and clinical features change with disease progression in COVID-19. With the worsening of the disease process, alveolar edema leads to increased right heart pressure, which in turn causes more tissue hypoxia and multiorgan failure [18].

Gattinoni et al conceptualized two distinct phenotypes: the "L" and "H" types (later stages/ARDS) at the ends of the clinical spectrum with possible intermediate cases with overlapping features $[16,17]$. The " $L$ " type is seen early on with low elastance/ high compliance, low lung weight, low VQ mismatch, and low recruitability. The lungs at this stage are compliant, can hold a good amount of air, are not affected by much edema; VQ ratio is due to defects in vasoregulation and perfusion, and since most of the lung is already aerated, there is not much scope for recruitability [16]. These patients have mild dyspnea, limited ground glass infiltrates on computed tomography (CT) scans, and they can withstand the distress. The " $\mathrm{H}$ " type is found later in the COVID-19 disease process with more resemblance to ARDS. It is the exact opposite of the "L" type with high elastance/low compliance, high lung weight, high VQ mismatch, and high recruitability [16]. These explain the resemblance to ARDS (noncardiogenic pulmonary edema, shunting, and decreased lung size for gas exchange). These patients are symptomatic and with extensive infiltrates on CT suggestive of alveolar edema, and increased risk of ending up on the ventilator support device. Based on these concepts, respiratory support in COVID-19 should focus on optimizing oxygenation, reducing pulmonary and vascular stress, preventing edema and lung injury, and recruitment of functional lung units [17].

For acute hypoxemic respiratory failure, the recommended goal is to provide supplemental oxygen aiming for oxygen saturation $\left(\mathrm{SpO}_{2}\right)$ 90-96\% [15]. Oxygen supplementation could be increased to considerably safer limits of $6 \mathrm{~L} / \mathrm{min}$ through a nasal cannula and $10 \mathrm{~L} / \mathrm{min}$ through a non-rebreathing mask. If conventional oxygen therapy fails, high flow nasal cannula (HFNC) is preferable to non-invasive ventilation (NIV) due to an increased risk of aerosolization with NIV. Early intubation is preferred, but the approach is controversial. During intubation, bag-valve-mask ventilation should be avoided, and the two-person technique is preferred [15]. Overall, the decision to initiate NIV, HFNC or intubation, should be made by balancing the risks and benefits to the patient, the risk of exposure to 
healthcare workers, and best use of local resources; this approach should be reassessed as new research data and resources become available. In a systematic review of 10 retrospective cohort studies which evaluated transmission of SARS-CoV to healthcare workers, endotracheal intubation had the highest risk (odds ratio (OR): 6.6; 95\% confidence interval (CI): 2.3 - 18.9), followed by non-invasive ventilation (OR: $3.1 ; 95 \%$ CI: 1.4 - 6.8), tracheostomy (OR: 4.2 ; 95\% CI: 1.5 - 11.5), and bag-mask ventilation [19]. In general, indications for intubation include clinically worsening respiratory distress, rapidly progressive disease, $\mathrm{SpO}_{2}$ less than $90 \%$ despite maximal supplemental oxygen, acidosis with $\mathrm{pH}$ less than 7.3, and partial pressure of carbon dioxide $\left(\mathrm{PaCO}_{2}\right)$ more than $50 \mathrm{~mm} \mathrm{Hg}$, multi-organ failure and hemodynamic instability.

The general guideline to initial ventilator settings includes the assist control (AC) mode with a tidal volume $4-8 \mathrm{~mL} / \mathrm{kg}$ ideal predicted body weight; respiratory rate 25 - 30 breaths/min; positive end expiratory pressure (PEEP) $10-15 \mathrm{~cm} \mathrm{H}_{2} \mathrm{O}$; target $\mathrm{SpO}_{2}$ 90-96\%; plateau pressure less than $30 \mathrm{~cm} \mathrm{H}_{2} \mathrm{O}$. The L type patients tolerate lower PEEP $\left(<10 \mathrm{~cm} \mathrm{H}_{2} \mathrm{O}\right)$ and higher tidal volume $(7-9 \mathrm{~mL} / \mathrm{kg})$. In these patients, higher PEEP redirects blood flow and creates dead space, while higher tidal volumes for hypercapnia are well tolerated due to high compliance. The H type patients, with low lung compliance, need higher PEEP $(<15 \mathrm{~cm}$ $\left.\mathrm{H}_{2} \mathrm{O}\right)$ and lower tidal volumes $(5-7 \mathrm{~mL} / \mathrm{kg})[17]$.

Prone positioning is an option when patients on ventilators have worsening oxygenation with mechanical ventilation (e.g., $\mathrm{PaO}_{2}$ /fraction of inspired oxygen $\left(\mathrm{FiO}_{2}\right)$ less than 150 $\mathrm{mm} \mathrm{Hg}$ for $12 \mathrm{~h}$ ). Patients must be maintained in a prone position for $12-16 \mathrm{~h}$ a day [15]. Contraindications to prone positioning include shock, active bleeding, recent tracheal surgery, multiple fractures, and spine instability [15]. Recruitment maneuvers, high PEEP strategies, and trial of inhaled pulmonary vasodilators are probable options for patients that failed prone positioning $[15,20]$. Neuromuscular blockade can be employed for patients with refractory hypoxemia on ventilator dyssynchrony (intermittent boluses are preferred unless persistent dyssynchrony) [15].

In general, extracorporeal membrane oxygenation (ECMO) is used in patients with refractory cardiac and respiratory failure in whom usually the venous blood is removed from the body and pumped through an artificial membrane lung, and is essentially a modified cardiopulmonary bypass. This oxygenation is needed for circulatory support and organ function. The role of ECMO in COVID-19 is unclear but may be used in patients with refractory hypoxemic respiratory failure. Venovenous ECMO has been used as a last resort for patients that have failed all other means; however, the mortality of COVID-19 patients who lands up on ECMO is extremely high and $>90 \%$ in one analysis [21].

\section{Pharmacological Therapy}

Multiple pharmacotherapies have been tried on an experimental basis on COVID-19 patients across the globe. Some studies claimed faster clearance of virus from the patient, and some demonstrated decreased mortality. No large randomized scale-controlled trial has settled the argument of the true effec- tiveness of any medicine against COVID-19. In this section, we will briefly present the proposed mechanism of action of individual medications that have been most commonly tried and trialed in recent times against COVID-19, and also discuss possible pros and cons of experimental use of such medications in these vulnerable patients. We have also provided an updated list of ongoing trials of most major medications that are being investigated as a possible effective pharmacological therapy against COVID-19 as a supplement to this article (Supplementary Material 1, www.jocmr.org).

\section{Hydroxychloroquine/chloroquine and azithromycin}

\section{Mechanism of action (MOA)}

Hydroxychloroquine and chloroquine block entry of the virus into human cells by proteolytic processing, inhibiting glycosylation of host receptors, and acidification inside endosomes. These agents may have immunomodulatory effects through blockage of autophagy and lysosomal activity in host cells, along with dissipating cytokine production and inhibition.

Azithromycin is an antibacterial macrolide and works through binding to 50s ribosomal subunit and inhibition of messenger RNA directed polypeptide synthesis. Antiviral mechanism of macrolides is scarce and was hypothesized to inhibit respiratory syncytial virus through the reduced expression of fusion protein receptor, activated isoform A of the Ras homologous (Rho) family, and the inhibition of subsequent Rho-kinase activation in human airway epithelial cells [22]. Azithromycin was shown to inhibit replication in Zika and Ebola viruses [23, 24].

\section{Pros and cons}

Chloroquine and hydroxychloroquine are relatively well tolerated and has been used for ages in patients with systemic lupus erythematosus (SLE) and malaria. However, both agents can cause serious adverse effects $(<10 \%)$, like hypoglycemia, retinopathy, psychiatric effects, QTc prolongation. Azithromycin is a commonly used macrolide for respiratory bacterial infections. Gautret et al concluded that combination therapy with azithromycin and hydroxychloroquine cured $100 \%$ of patients virologically on day 6 compared to $57.1 \%$ in patients treated with hydroxychloroquine only, and $12.5 \%$ in the control group $(\mathrm{P}=0.001)$ [25]. However, the risk of QT prolongation from these two drugs should be considered, and caution should be taken, especially in cardiac patients, while administering this combination [26]. Moreover, a study among 368 USA veterans found no benefit, rather touted hydroxychloroquine to be more harmful due to its side effect profile [27].

\section{Proposed dose for COVID-19}

Hydroxychloroquine dose most used is $400 \mathrm{mg}$ twice daily orally for two doses, then $400 \mathrm{mg}$ daily orally for a total of 5 days. Chloroquine dose suggested by FDA is $1 \mathrm{~g}$ on day 1 , then 
$500 \mathrm{mg}$ daily for 4 - 7 days total.

\section{Remdesivir}

$M O A$

Remdesivir, or GS-5734, is an adenosine monophosphate prodrug that metabolizes to an active $\mathrm{C}$-adenosine nucleoside triphosphate analog, thereby interfering with the action of viral RNA-dependent RNA polymerase.

\section{Pros and cons}

The agent was first discovered in 2015 in the process of finding antimicrobials with activity against RNA viruses. Initially, it was used for Ebola treatment. It has shown promising results in animals' studies with MERS and SARS caused by a coronavirus.

Remdesivir received EUA by FDA last week for use in patients hospitalized with COVID-19 based on a clinical trial, which showed remdesivir, accelerated the recovery time by $31 \%$, from 15 days to 11 days in patients who received treatment with it. It reduced the mortality from $11 \%$ to $8 \%$, but it was not statistically significant [28]. Few case reports and series suggesting its effectiveness in the novel COVID-19 has been published [29]. Notable side effects are nausea, vomiting, and reversible rise in aspartate aminotransferase and alanine transaminase.

\section{Proposed dose for COVID-19}

The current dose under investigation is a single $200 \mathrm{mg}$ loading dose, followed by $100 \mathrm{mg}$ daily infusion [29]. Under this EUA, the recommended dosing duration for patients requiring invasive mechanical ventilation and/or ECMO, and for patients not requiring invasive mechanical ventilation and/or ECMO is 10 days and 5 days, respectively [28]. Therapy is not recommended in patients with an estimated glomerular filtration rate less than $30 \mathrm{~mL} / \mathrm{min}$ [29].

\section{Favipiravir}

\section{$M O A$}

Favipiravir inhibits influenza viral replications by targeting RNA polymerase, and this mechanism is also being applied to the novel coronavirus, which is a single-stranded RNA virus and requires RNA polymerase for replication [29].

\section{Pros and cons}

Favipiravir is not available commercially in the USA. The safety and efficacy of the drug is not established as of now. Favipiravir is a generic version of brand Avigan used for treating novel influenza infections in Japan. Notable side effects include decreased neutrophil count, diarrhea, increased uric acid levels, elevated transaminases [29].

\section{Proposed dose for COVID-19}

Recommended dosing is 2,400 to $3,000 \mathrm{mg}$ loading dose every $12 \mathrm{~h}$ for two doses, followed by 1,200 to $1,800 \mathrm{mg}$ twice a day as maintenance dose [29].

\section{Interleukin (IL)-6 pathway inhibitor}

\section{$M O A$}

Elevated levels of the inflammatory marker, including IL-6, were found in the blood of COVID-19 patients, and were reported to have a bad prognosis in patients. IL-6 is a proinflammatory cytokine and binds to both soluble IL-6 receptor (sIL-6R) and membrane-bound IL-6R (mIL-6R). The resulting complex activates an inflammatory response through interaction with transducing component glycoprotein 130 (gp130), which can result in a cytokine storm [30]. Sarilumab and tocilizumab are the two IL-6 inhibitors widely available in the markets, and they bind specifically to sIL-6R and mIL-6R, and block signal transduction.

\section{Pros and cons}

Cytokine storm in response to COVID-19 has been found to have devastating consequences in critically ill patients and may facilitate shock and multi-organ failure. IL-6 inhibitors can be helpful by diminishing the effect of an overactive cytokine system.

New-onset abdominal symptoms should be monitored as there were reported cases of GI perforation, specifically in patients with a history of diverticulosis. Baseline lipid panel and liver function testing should also be done as these drugs might elevate these parameters significantly.

\section{Proposed dose for COVID-19}

Standard dosing for these medications has been used for experimental purposes.

\section{Lopinavir/ritonavir}

\section{$M O A$}

The lopinavir component binds to the site of viral protease activity and inhibits the cleavage of viral Gag-Pol polyprotein 
precursors into individual functional proteins required for infectious human immunodeficiency virus (HIV). This results in the formation of immature, noninfectious viral particles. The ritonavir component inhibits the cytochrome P450 3A (CYP3A) metabolism of lopinavir, allowing increased plasma levels of lopinavir.

\section{Pros and cons}

Widely and successfully used in HIV management, this combination has been tried in the management of 2019 novel coronavirus (2019-nCoV). This drug has shown some effect in the in vitro model for MERS and SARS treatment [31, 32].

Clinical trials so far have not managed to show any benefit of this combination in the treatment. In a recently published article from a study in China, this combination has not shown any effective benefit. No significant differences in viral clearance or 28-day mortality rates were observed in 199 studied patients [33]. Adverse reactions should be kept in mind. The most frequently reported reactions in patients receiving lopinavir therapy are asthenia, diarrhea, and nausea. Elevated total bilirubin, hepatic enzyme levels, and triglycerides have also been reported [34].

\section{Proposed dose for COVID-19}

Commonly studied lopinavir/ritonavir dosing in COVID-19 patients is $400 \mathrm{mg} / 100 \mathrm{mg}$ twice daily for up to 14 days.

\section{Histamine 2 receptor antagonist (H2RA)}

\section{$M O A$}

Histamine has pleiotropic effects on the immune system from different natures of its receptors. One of its effects being immunomodulation resulting in sepsis was noticed in diabetic mice. This happens through decreased neutrophil recruitment and impaired oxidative burst from elevated histamine levels $[35,36]$. H2RAs block these immunosuppressive effects of histamine and stimulate the functions of T and B white cells [37]. The antiviral effects of this H2RA were demonstrated in patients with herpes zoster infection, herpes simplex virus (HSV), and human papillomavirus (HPV) [38-40]. It was also shown that cimetidine, an H2RA, increased immunogenicity when given as an adjuvant along with HBV viral vaccines [41]. It also decreased HIV replication in vitro [42].

\section{Pros and cons}

Although H2RA is a very commonly used medication that is even available over the counter, no conclusive data are supporting how H2RA helps against COVID-19. This thought originated from Michael Callahan, an infectious disease doctor at Massachusetts General Hospital. He observed that many of the COVID-19 survivors had chronic heartburn and took famotidine rather than omeprazole, which is more expensive when he was working in Wuhan during the coronavirus epidemic began. It was later investigated in more than 6,000 patients who recovered and found a slightly higher number in the famotidine group, but was not high enough to be statistically significant.

\section{Proposed dose for COVID-19}

Standard dose to treat gastroesophageal reflux disease.

\section{Interferon (IFN) beta}

\section{$M O A$}

IFN-beta is a subtype of type I IFN secreted by many cell types, mostly by plasmacytoid dendritic cells upon recognition of viral components by pattern recognition receptors (PRR). IFN-stimulated genes (ISG) are involved in inflammation and immunomodulation. ISGs interfere with viral replication and viral spread through different pathways like cytokine secretion or slowdown of cell metabolism [43].

\section{Pros and cons}

Data obtained from the experiments involving treatment of SARS-CoV and MERS-CoV and ISG's ability to disrupt the IFN signaling pathway would be valuable for selecting IFNbeta as a potential treatment option against SARS-CoV-2 [44, 45].

Type I (IFN-alpha and beta) IFNs were efficient in vitro and also in specific animal models but failed to control the disease in humans [44]. It was hypothesized that SARS-CoV-2 induces an IFN-I mediated antiviral response, leading to tissue damage.

\section{Proposed dose for COVID-19}

IFN-beta is the most relevant IFN-I that should be given as early as possible to optimize antiviral therapy and avoid complications from the virus [46]. No specific dose has been validated, especially for COVID-19. The general dosing guideline is being followed.

\section{Convalescent plasma (CP)}

CP or immune plasma, is the plasma collected from donors who have successfully survived an infectious disease by generating antibodies. CP has been in use for over 100 years to treat a variety illness starting with measles, polio, chickenpox to recent epidemics as SARS-CoV-1 epidemic (SARS) in 2003, H1N1 influenza pandemic (H1N1) in 2009 - 2010, avian 
influenza A (H5N1), Ebola and MERS-CoV epidemic in 2012 [47-52].

\section{$M O A$}

The antibodies in the CP could potentially limit the viral replication, can mediate cellular toxicity and/or phagocytosis, and the plasma components can exert vital clinical effects such as replacing the coagulation factors, complement activation. Also, CP may potentially offer the only short-term strategy to confer immediate immunity to infection susceptible patients [53]. In the absence of an effective specific treatment for COVID-19, clinicians across the globe have used the $\mathrm{CP}$ with varying success $[54,55]$.

\section{Pros and cons}

During the 2005 SARS outbreak in Hong Kong, Cheng et al [56], have published the most extensive study on the outcomes of 1,775 patients with the infection, of whom, 80 patients received $\mathrm{CP}$ had a lower mortality rate $(12.5 \%)$ compared to overall SARS-related mortality. The study was not a randomized trial, and no adverse events reported [56]. Shen et al [54] have published a preliminary study of five patients with COVID-19 who were severely ill and treated with CP from China. All five patients were mechanically ventilated, and one needed ECMO. The donor CP, an apheresis product, had demonstrable immunoglobulin $\mathrm{G}(\mathrm{IgG})$ and $\operatorname{IgM}$ anti-SARS$\mathrm{CoV}-19$ antibodies and in vitro virus-neutralizing properties. The authors concluded that the $\mathrm{CP}$ might have contributed to the recovery, although the patients were also on lopinavir/ritonavir antiviral therapy and IFN [57].

Despite the numerous case series and emerging evidence with $\mathrm{CP}$ is compelling and well documented, it has several limitations as it is not evaluated in a randomized clinical trial, to determine the actual clinical benefit when compared to those who have not received it $[53,58]$. In almost all the case series and studies published so far, the patients received numerous other therapies, including various antivirals, steroids, etc. It is also interesting to note that the timing of administration of the $\mathrm{CP}$ was different in multiple studies. Prior studies have shown that passive antibody therapy is most effective if given prophylactically or used early in the disease course.

\section{Proposed dose for COVID-19}

Even though there is no compelling evidence from large scale randomized trials, the FDA has begun allowing $\mathrm{CP}$ to be used in patients with severe or immediately life-threatening COVID-19 infections starting March 24, 2020. The treatment with $\mathrm{CP}$ is considered experimental. It is important to note that effective formulations such as a convalescent plasma or H-Ig or immunoglobulins, is still unknown. A person who has tested positive for COVID-19 and recovered with no symptoms for 14 days could be a potential donor in the presence of high enough antibody levels in the plasma and negative for possible infections such as HIV, hepatitis C, etc. The donor and the recipient should have compatible blood groups.

Though been in use for over 100 years for various infectious diseases, there is a severe lack of cooperative global efforts to use $\mathrm{CP}$ as initial therapies against the new and emerging epidemics and pandemics. More than 4.5 million global infections and growing, COVID-19 provides an opportunity to perform large-scale rigorous clinical studies on CP against the viral agents to establish clinical efficacy. This pandemic could provide a strategic pathway for future viral pandemic management with $\mathrm{CP}$.

\section{Plasma adsorption and exchange}

Emerging evidence suggests that managing cytokine storm in critically ill COVID-19 patients by using steroid or IL-6R blocking antibodies may be beneficial $[30,59]$. In the case of fulminant systemic infection, patients may develop sepsis, ARDS, and multi-organ failure, which are not unique to coronavirus. Treatment with effective antiviral therapy is being sought; however, treatment of the systemic response to this viral infection is likely to be equally or more important. Overzealous host response to infection has been well described and involves a complex interaction of cytokine storm, inflammation, endothelial dysfunction, and pathologic coagulation [6063 .

The utilization of blood purification therapy in the form of plasma adsorption or therapeutic plasma exchange (TPE) has been proven in the setting of sepsis [64, 65], but the same has not been adequately proven in critically ill COVID-19 patients. Knowledge of optimum management of critically ill COVID-19 patients in the late phase is quite limited. The presence of cytokine storm or pathogenic antibodies in critically ill COVID-19 patients has a strong correlation with the disease severities. Monitoring inflammation and antibodies is significant, especially in patients infected by the virus with persistent fever or abnormal coagulopathy. Expeditious control of the cytokine storm utilizing plasma adsorption or TPE might be beneficial to selective patients with COVID-19 [66]. These therapies are well tolerated if performed with the close guidance of specialists, and risks of infection or bleeding are minimized. So far, randomized trial data are not available regarding this in COVID-19, but such clinical trials should be done to utilize blood purification therapy to appropriate patients.

\section{Special Circumstances and Considerations}

\section{HIV and COVID-19}

There are close to 40 million people living with HIV globally. There are no major studies that have looked at clinical characteristics, antiretroviral treatment (ART), and outcomes in people with COVID-19 co-infected with HIV. Patients with HIV accounted for almost $1 \%$ of patients with COVID-19 who required admission to hospital in Barcelona during this pandemic. 
It is unclear if ART offers any protection from COVID-19 in HIV patients. However, there have been reports that patients living with HIV and COVID-19 pneumonia receiving ART may have moderate symptoms and faster improvement than the general population and atypical CT imaging features from the conventional population. The preliminary clinical trials showed no clinical benefit of lopinavir/ritonavir for COVID-19 [33]. In a recent clinical case series by Blanco et al, five patients with COVID-19 and HIV who were treated with protease inhibitors regimen recovered well with no deaths reported [67]. The efficacy of ART medications on COVID-19 needs to be further confirmed in future studies so that the management and prognosis of patients co-infected with HIV and COVID-19 might be improved [68].

\section{Patients with rheumatological conditions on immunosup- pression medications and COVID-19}

COVID-19 has raised various concerns among patients on chronic immunosuppressive therapy because the immune response to the virus was thought to be lowered. This has created a concern for possible non-adherence to these medications. Immunosuppressive agents are known to impair immune function, thereby increasing the risk of any infection. However, it has been described that uncontrolled disease activity in patients with rheumatoid arthritis and SLE puts them at a significantly higher risk for outpatient infections and hospitalization $[69,70]$. It is of utmost importance to optimize patient medication adherence to prevent any discontinuation of chronic treatment, which may lead to disease flare and increased risk of infection [71].

Some disease-modifying drugs which are commonly used to treat rheumatic diseases, such as biologics targeting IL-6 (tocilizumab, sarilumab) and IL-1 (anakinra), are being investigated as potential therapies for COVID-19, especially in patients with cytokine storm and reactive hemophagocytic lymphohistiocytosis. However, conclusive evidence is lacking to guide treatment decisions at this point.

Case series from Italy by Monti et al [70] looked at the clinical course of COVID-19 in a series of 320 patients with chronic arthritis treated with targeted immunosuppressive therapies. This included patients with rheumatoid arthritis and spondyloarthritis, treated with biological derived diseasemodifying anti-rheumatic drugs (bDMARDs), targeted synthetic disease-modifying anti-rheumatic drugs (tsDMARDs) or tumor necrosis factor inhibitors. bDMARDs or tsDMARDs were held in eight patients with symptomatic COVID-19 with fever and shortness of breath. The study reported no significant relapses of the rheumatic disease, no mortality from COVID-19. It is suggested that patients with chronic arthritis treated with bDMARDs or tsDMARDs are not at increased risk of respiratory or life-threatening complications from COVID-19 compared with the general population [70]. These findings are similar to the different coronavirus outbreaks, such as SARS and the MERS. There has been no increased mortality reported in patients undergoing immunosuppression for organ transplantation, cancer, or autoimmune diseases.

As we await more clinical studies and concrete data, immunosuppressed patients should continue to follow basic personal prevention procedures (e.g., social distancing, hand hygiene, and face mask use). At the same time, the clinicians should advocate against the discontinuation of chronic immunosuppressive agents by patients. Such disruption of therapy could lead to an increased risk of relapses and morbidity from chronic rheumatological conditions.

\section{Organ transplant patients and COVID-19}

It is thought that organ transplanted patients have reduced the severity of lung injury and atypical symptoms of COVID- 19 . Although transplanted patients could be more susceptible to COVID-19 infection with atypical manifestations, the chronic use of immunosuppressive drugs could potentially represent a "protective factor" for the severe clinical complication of the disease through excessive cytokine reaction. However, there has been conflicting data from different parts of the world on this subject.

A small case study by Li et al described COVID-19 infection in two solid-organ transplanted patients with variable symptoms who have survived the event [72]. It may be possible that the activation of the immune system, especially $\mathrm{T}$ cells, leads to typical diffuse alveolar injury in the lungs.

Although immunosuppressed solid-organ transplanted patients could be more susceptible to COVID-19 with severe clinical manifestations, the anti-inflammatory effects of immunosuppression could diminish the clinical expression of disease. Tacrolimus and cyclosporine are the most commonly used drugs for maintenance immunosuppression following solid-organ transplantation. They reduce the production of IL2 , a regulator of proliferation, survival, and maturation for all $\mathrm{T}$ cell populations [73]. Lymphopenia is observed in severe cases of COVID-19 that could be caused by lung sequestration of hyperactivated $\mathrm{T}$ cell, and immunosuppression medications in these patients could limit the effect and severity of lung injury.

On the contrary, another single-center case series from Spain by Fernandez-Ruiz et al [74] described their preliminary experience with 18 solid-organ transplant (kidney, liver, and heart) recipients diagnosed with COVID-19. Fever (83.3\%) and radiographic abnormalities in the form of unilateral or bilateral/multifocal consolidations (72.2\%) were the most common presentations. They reported that the case fatality rate was $27.8 \%(5 / 18)$ and which suggested that COVID-19 has a severe course in solid-organ transplant patients.

More data from a recent study published by Akalin et al [75] has shown significant mortality in kidney transplant patients. The study identified 36 consecutive adult kidney transplant recipients who tested positive for COVID-19. They concluded that kidney transplant recipients with COVID-19 had less fever as an initial symptom, lower cluster of differentiation 3 (CD3), CD4, and CD8 cell counts, and more rapid clinical progression than persons with COVID-19 in the general population. These patients had very low CD3, CD4, and CD8 cell counts indirectly support the need to decrease doses of immunosuppressive agents in patients with COVID-19, especially in those who have recently received antithymocyte globulin, which decreases all T-cell subsets for many weeks. The study 
reported extremely high early mortality, $28 \%$ at 3 weeks as compared with the usual $2-5 \%$ mortality in the general population. In this limited cohort of kidney transplant patients, COVID-19 induced pneumonia is characterized by a high risk of progression and significant mortality. Hopefully, more studies in the future will guide us with the management strategy of COVID-19 infection in solid-organ transplant patients and immunosuppression medications.

\section{Pregnancy and COVID-19}

The comprehensive impact of COVID-19 infection on a pregnant woman is currently unclear. International Federation of Gynecology and Obstetrics (FIGO) has suggested a few important guidelines until more definitive data become available [76]. There are several concerns and questions related to complications during pregnancy and the potential effect on fetal and neonatal outcomes. Hence, pregnant women require special attention concerning prevention, diagnosis, and management [77].

Comorbidities like hyperglycemia and hypertension should be carefully managed with existing protocols as in routine pregnancy. COVID-19 infection itself is not an indication for delivery unless maternal health is severely jeopardized. Timing and mode of delivery should be individualized, depending mainly on the clinical status of the patient, gestational age, and fetal condition. Currently, there is no evidence that COVID-19 infection is associated with fetal or placental complications or risk of congenital malformation. The treatment approach depending on the severity of COVID-19 infection is the same as for confirmed, suspected, and probable cases in the general population. In severe cases, the patient should be managed with the support of a multidisciplinary team (obstetricians, intensivists, internists, neonatologists, infectious disease specialists). Currently, there is no proven antiviral treatment for COVID-19 patients, although antiretroviral drugs are being investigated on patients with severe symptoms. A chest CT scan may be included in the workup of pregnant women, if necessary, with suspected/probable/confirmed COVID-19 infection after discussing the risk and benefits with the patient.

Data from five small series, including 56 pregnant women diagnosed with COVID-19 during the second and third trimester, demonstrated that most common symptoms at presentation were fever and cough. Two-thirds of patients had lymphopenia and increased C-reactive protein (CRP), and $83 \%$ of cases had a chest CT scan showing multiple patches of ground (glass opacity in the lungs). The study by Chen et al looked into details of nine live births in patients with COVID-19 suggested there was no evidence of vertical transmission in late pregnancy. There is currently insufficient evidence regarding the safety of breastfeeding and the need for mother/baby separation [78, 79]. If the mother is severely ill, the separation appears the best option, with attempts to express breast milk to maintain milk production. If the patient is asymptomatic or mildly affected, breastfeeding and rooming-in can be considered by the mother in coordination with healthcare providers.

Long-term outcomes of pregnant patients with COVID-19 are yet to be determined. COVID-19 related to maternal, fetal, and neonatal outcomes will need further studies for more evidence-based management of pregnant women and neonates.

\section{COVID-19 in end-stage kidney disease (ESKD) patients receiving chronic dialysis}

ESKD patients likely are at significant risk of COVID-19 infection due to suppression of the immune system, and may have poorer outcomes from COVID-19 for the conglomeration of risk factors in individual patients. Patients treated in outpatient dialysis centers present a challenge as the risks of transmission to the medical staff, and other patients are high due to treatment in the same confined space. If infected, the intensity of dialysis requiring specialized resources poses a significant burden to the patients and the medical community. Hence, preventive measures are most critical to reduce the incidence of infection in this vulnerable population. Strict protocols have been placed to minimize the spread of this highly contagious COVID-19 as it is well-known from previous epidemics that the mortality is much higher in dialysis patients compared with the general population.

A dialysis team consisting of nephrologists, nursing staff, dieticians, and social workers are playing a critical role in the prevention and containment of COVID-19. Staff members have been particularly educated on guidelines to minimize the infection risk and also to manage patients with signs of infection. CDC has recommended guidelines to keep the dialysis units safe to continue treatment at the dialysis unit itself without placing a burden on the hospitals, which are already overwhelmed with the COVID-19 cases. Sick members of the team are advised to stay home. Patients are instructed to stay at home while off dialysis. Screening of each individual that arrives at the dialysis unit using a questionnaire and temperature check has been implemented. Early recognition and isolation of individuals with a respiratory infection, fever, cough, and upper airway involvement have been critical. Aggressive disinfectant strategies and universal mask usage policies have been implemented. Patients with confirmed COVID-19 infection are admitted to cohort dialysis units if they are asymptomatic or with mild symptoms. Patients with moderate to severe symptoms are admitted to the hospital for isolation and treatment $[80,81]$. Patients receiving home dialysis (peritoneal and home hemodialysis (HD)) are assisted using telehealth to prevent any COVID-19 exposure. In light of the ongoing pandemic, the advantage of home dialysis modalities is evident from the perspective of minimal exposure risk. However, home dialysis patients, including peritoneal dialysis (PD) patients, can also contract this viral infection [82].

One case series from a HD center in Wuhan reported COVID-19 infection in 37 out of $230 \mathrm{HD}$ patients $(16.1 \%)$ and four out of 33 staff members (12.1\%). Dialysis patients were noted to have lymphopenia, lower serum levels of inflammatory cytokines, and a milder clinical disease than the other patients. Seven out of 33 COVID-19 dialysis patients died during the pandemic, and it was presumed that the deaths were not directly secondary to COVID-19, but due to cardiovascular and hyperkalemia. The outcome of the four staff members with COVID-19 was uneventful [83]. 
The AKI incidence in COVID-19 ranged from 3-15\% on literature review, and the incidence has significantly increased from $15 \%$ to $50 \%$ in severe infection, often needing intensive care unit (ICU) admission and renal replacement therapy (RRT). AKI is an independent risk factor for mortality in COVID-19 patients. AKI is a prominent complication in critically ill COVID-19 patients. It may warrant specific strategic management plans, including the variation of RRT modalities (e.g., continuous RRT, prolonged intermittent RRT, conventional HD) to optimize the appropriate use of limited dialysis resources [81]. The pandemic is still evolving, and we expect that the morbidity and mortality may be much different in different geographical areas, which will partly depend on the patient population, health care availability, and protocols followed in those places. Much larger studies with robust data will help us understand the impact of COVID-19 on ESKD patients receiving dialysis and help reduce mortality.

\section{Discontinuation of Isolation Precaution and "Lockdown" in the Community}

In the last 100 days, the global pandemic of COVID-19 has so far infected more than 3 million people worldwide and led to the death of over 200,000. Many countries are under a lockdown of some form. This has caused severe disruption with devastating social, economic, and health care consequences. Countries are struggling to find ways to get back to the normalcy of some degree and are preparing for the next wave of infection to impact in the fall and winter of 2020.

Countries, where community transmission has led to more significant outbreaks and subsequent exponential growth in cases and mortality, have introduced physical distancing measures. These measures are in the forms of "shutdowns" and "lockdowns" that have socially and economically impacted everyone but disproportionately affected poor, migrant and disadvantaged groups leading to significant mortality and morbidity in these groups. Although detailed peer-reviewed reports regarding these economic and care delivery consequences have not been published, publicly available information in reputable media indeed points towards the above consequences.

Arguments are being made that the economic costs of social distancing and stay-at-home are worse than the medical catastrophe. However, in the absence of widely available testing facilities, proven pharmacotherapeutics, and the introduction of an effective vaccine, mere lifting of social distancing restrictions will not be effective in ending the economic damage or slow down, as the vast majority of fearful people will not participate in the economic activity. As can be seen during this devastating pandemic, public health and economic goals are mutually exclusive.

By the beginning of May 2020, hundreds of clinical trials are being conducted worldwide to find effective pharmacotherapy and develop a vaccine to conquer SARS-CoV-2, which was summarized here (Supplementary Material 1, www.jocmr.org) [84]. Approximately 12 clinical trials with therapeutic intervention are completed and results are pending for most of them apart from the clinical trial from Hong Kong which published results recently. A phase 2 clinical trial involving IFN beta- $1 \mathrm{~b}$, lopinavir/ritonavir, mainly used for the treatment of HIV, ribavirin mainly used in combination with IFN for the treatment of hepatitis $\mathrm{C}$ was tested in 86 patients for 14 days for treatment of COVID-19 in Hong Kong. The treatment group took all the above medications while the control group took lopinavir/ritonavir. The patient is in the active intervention group, and the control group had viral clearance at an average of 7 days and 12 days, respectively. The combination of medications was well tolerated, with no severe side effects [85].

An effective vaccine is the only true remedy for this pandemic, but the manufacturing of such a vaccine may well take at least 12 to 18 months to develop. In the meantime, there is always the concern that the virus may mutate. This means we must prepare ourselves to sustain without a vaccine or effective treatment for months.

It is hard to estimate the costs of management and pharmacotherapy for COVID-19 as the cost significantly varies from country to country, including hospital management as well as the cost of the drugs. As per the article from Forbes, the recently approved remdesivir, it could cost up to $\$ 4,500$ for a course of treatment, which could still be cost-effective as per the institute of clinical and economic review. The manufacturers of therapeutics of COVID-19 might adopt a different pricing model, including some companies on a not-for-profit basis [86]. A review by Hill et al [87] estimated minimum costs of production from the active pharmaceutical ingredient's costs. Remdesivir, which is approved for EUA by FDA costs $\$ 0.93$ / day. Other drugs under a further investigation like favipiravir costs $\$ 1.45 /$ day, hydroxychloroquine $\$ 0.08 /$ day, azithromycin $\$ 0.10 /$ day, lopinavir/ritonavir $\$ 0.39 /$ day. If the repurposed drugs demonstrate efficacy agonist COVID-19, as per the above, they could be manufactured at lower costs [87].

The authors of this article have decided to dedicate this section to delve into ways how we can safely go back to socalled normal life, which may look vastly different from preCOVID times. WHO has proposed a Strategic Preparedness and Response Plan (SPRP) on the phased transition from widespread transmission to a low level or no transmission and practical guidance on how to effectively manage broader social, humanitarian, and economic impacts of COVID-19 [88].

These measures can be divided into the following broad categories:

\section{Slowing down the transmission of disease and providing care for severely sick ones}

This is to ensure the appropriate utilization of limited healthcare resources and to avoid overwhelming the global healthcare infrastructures.

\section{Ramping up testing for infection and antibody testing to assess the level of viral prevalence and immunity}

So, resources can be allocated to high-risk areas. Availability of fast, reliable point of care testing capability would be able 
to identify and avoid transmission of this virus even by asymptomatic carriers. For example, currently, all routine surgical procedures are postponed across the US healthcare systems. However, the availability of such testing capacity may be implemented to screen the patients and surgical team preoperative to ascertain their infection status and go ahead with even routine surgical procedures, which make the bulk of the revenues for hospitals and it is extremely important for the sustainability of any healthcare system.

\section{Individual, community level, governmental, and private sector engagement}

A massive coordination is necessary for the successful "reopening" of businesses in a way so that the public health is not put at high risk and economic activities can be improved. Prolonged inactivity of business sectors and industries will have catastrophic effects on the economy in general and will have far-reaching consequences on the earning, health, and life of people.

Many proposals have been put forth by organizations such as the American Enterprise Institute, the Center for American Progress, and Harvard University's Edmond J. Safra Center for Ethics [89, 90]. The recommendations have a common theme and can be summarized as follows.

The first step is to keep the infection rates low by imposing strict social distancing measures. Meanwhile, we need to significantly ramp up testing capabilities so that when we are ready to ease restrictions, anyone who needs a test can be tested for infection as well as antibodies that would identify who are immune. Second, when it is time to relax the social distancing, people with immunity could be provided with "immunity passports" utilizing digital approval pass, and could be allowed to move freely and get back in the active workforce. People who test negative for immunity may be allowed to move freely and work; however, they will need to be tracked to make sure they are not in close contact with people with suspected or active infection. The tracking has been done by some countries using peer-to-peer tracking apps such as trace together and covidnearyou.org so that we eliminate the need for centralized datakeeping and protect privacy and civil liberties of the public.

There remain significant hurdles to the above strategy. After several weeks of strict lockdown, many states in the USA no longer has stay-at-home orders, testing capacity improved significantly but antibody testing remained scarce and its interpretation - uncertain. Negative economic, psychological and civil impacts on the population in general due to lock-down of several weeks became evident despite federal financial aid for the public and businesses in USA. There is speculation of "second wave" of pandemic with the lockdown being lifted off, as has been suggested possible from preliminary data available from South Korea and Japan with similar approach. Unfortunately, the influence of "lock-down fatigue" is undeniable and desperation of communities to get back to normal routine is palpable. There was decrease in the daily death rates due to COVID-19 in USA by end of May 2020. However, in order to continue to curb down the toll of the pandemic, we need to continue active contact tracing, isolation of positive patients, judicious use of emerging medical therapy, as appropriate. A seamless coordination of data gathering, strategy making and execution of plan is essential and teamwork between all relevant governmental agencies is of paramount importance in this testing times. Clear, consistent and unbiased guidance from trusted authorities to keep the general public well-informed and compliant is crucial, too. Ultimately, it will certainly take the effort of the people of this world to overcome the sinister grip of this pandemic on our lives, health and wellbeing.

\section{Supplementary Material}

Suppl 1. Summary of Multiple Clinical Trials Available All Over the World on COVID-19.

\section{Acknowledgments}

None to declare.

\section{Financial Disclosure}

None to declare.

\section{Conflict of Interest}

None to declare.

\section{Author Contributions}

$\mathrm{SB}$ is the first and principal author of this article. All other authors contributed to sub-sections of the article. VM is responsible for creating the supplementary table and complete review of the manuscript. All the authors reviewed the manuscript and agreed with the findings and interpretation.

\section{Data Availability}

The authors declare that data supporting the findings of this study are available within the article.

\section{Abbreviations}

WHO: World Health Organization; 2019-nCoV: 2019 novel coronavirus; COVID-19: coronavirus disease 2019; SARS-CoV-2: severe acute respiratory syndrome coronavirus 2; FDA: Food and Drug Administration; MERS: Middle Eastern respiratory syndrome; AKI: acute kidney injury; ESKD: end-stage kidney disease; ACEI: angiotensin-converting enzyme inhibitor; RNA: ribonucleic acid; ICU: intensive care unit; ARDS: acute respiratory distress syndrome; CT: computed tomography; ECMO: extracorporeal membrane oxygenation; CDC: Centers for Dis- 
ease Control and Prevention; PPE: personal protective equipment; NSAIDS: non-steroidal anti-inflammatory drugs; SPRP: Strategic Preparedness and Response Plan; HD: hemodialysis; PD: peritoneal dialysis; RRT: renal replacement therapy; FIGO: International Federation of Gynecology and Obstetrics; CRP: Creactive protein; HCP: health care personnel; ARB: angiotensin receptor blocker; H2RA: histamine 2 receptor antagonist; IL: interleukin; IFN: interferon; AC: assist control; PEEP: positive end expiratory pressure; NIV: non-invasive ventilation; HFNC: high flow nasal cannula; PAPR: powered air-purifying respirator; $\mathrm{CP}$ : convalescent plasma; VQ: ventilation-perfusion; HIV: human immunodeficiency virus

\section{References}

1. https://www.cdc.gov/coronavirus/2019-ncov/cases-updates/cases-in-us.html.

2. World Health Organization. https://www.who.int/emergencies/diseases/novel-coronavirus-2019. Accessed on June 1, 2020.

3. Day M. Covid-19: ibuprofen should not be used for managing symptoms, say doctors and scientists. BMJ. 2020;368:m1086.

4. WHO. The use of non-steroidal anti-inflammatory drugs (NSAIDs) in patients with COVID-19. https://www.who. int/publications-detail/the-use-of-non-steroidal-anti-inflammatory-drugs-(nsaids)-in-patients-with-covid-19.

5. Russell CD, Millar JE, Baillie JK. Clinical evidence does not support corticosteroid treatment for 2019-nCoV lung injury. Lancet. 2020;395(10223):473-475.

6. Wu C, Chen X, Cai Y, Xia J, Zhou X, Xu S, Huang H, et al. Risk factors associated with acute respiratory distress syndrome and death in patients with coronavirus disease 2019 pneumonia in Wuhan, China. JAMA Intern Med. 2020.

7. Tang N, Li D, Wang X, Sun Z. Abnormal coagulation parameters are associated with poor prognosis in patients with novel coronavirus pneumonia. J Thromb Haemost. 2020;18(4):844-847.

8. Zhou F, Yu T, Du R, Fan G, Liu Y, Liu Z, Xiang J, et al. Clinical course and risk factors for mortality of adult inpatients with COVID-19 in Wuhan, China: a retrospective cohort study. Lancet. 2020;395(10229):1054-1062.

9. Centers for Disease Control and Prevention. People who are at higher risk for severe illness https://www.cdc.gov/ coronavirus/2019-ncov/need-extra-precautions/peopleat-higher-risk.html. Accessed on April 1, 2020.

10. Statement from the American Heart Association, the Heart Failure Society of America and the American College of Cardiology. Patients taking ACE-i and ARBs who contract COVID-19 should continue treatment, unless otherwise advised by their physician. https://newsroom. heart.org/news/patients-taking-ace-i-and-arbs-who-contract-covid-19-should-continue-treatment-unless-otherwise-advised-by-their-physician.

11. Yuan S. Statins may decrease the fatality rate of middle east respiratory syndrome infection. mBio. 2015;6:e01120.

12. The American Academy of Dermatology. https://assets.
ctfassets.net/1ny4yoiyrqia/PicgNuD0IpYd9MSOwab47 /023ce3cf6eb82cb304b4ad4a8ef50d56/Biologics_and_ COVID-19.pdf.

13. Guan WJ, Ni ZY, Hu Y, Liang WH, Ou CQ, He JX, Liu L, et al. Clinical characteristics of coronavirus disease 2019 in China. N Engl J Med. 2020;382(18):1708-1720.

14. Centers for Disease Control and Prevention. Interim clinical guidance for management of patients with confirmed 2019 novel coronavirus (2019-nCoV) infection, U.F. 2020. https://www.cdc.gov/coronavirus/2019-ncov/hcp/ clinical-guidance-management-patients.html. Accessed on April 29, 2020.

15. Alhazzani W, Moller MH, Arabi YM, Loeb M, Gong MN, Fan E, Oczkowski S, et al. Surviving Sepsis Campaign: guidelines on the management of critically ill adults with Coronavirus Disease 2019 (COVID-19). Intensive Care Med. 2020;46(5):854-887.

16. Gattinoni L, Chiumello D, Caironi P, Busana M, Romitti F, Brazzi L, Camporota L. COVID-19 pneumonia: different respiratory treatments for different phenotypes? Intensive Care Med. 2020.

17. Marini JJ, Gattinoni L. Management of COVID-19 Respiratory Distress. JAMA. 2020.

18. Luks AM, Freer L, Grissom CK, McIntosh SE, Schoene RB, Swenson ER, Hackett PH. COVID-19 lung injury is not high altitude pulmonary edema. High Alt Med Biol. 2020.

19. Tran K, Cimon K, Severn M, Pessoa-Silva CL, Conly J. Aerosol generating procedures and risk of transmission of acute respiratory infections to healthcare workers: a systematic review. PLoS One. 2012;7(4):e35797.

20. Pan C, Chen L, Lu C, Zhang W, Xia JA, Sklar MC, Du $B$, et al. Lung Recruitability in COVID-19-associated Acute Respiratory Distress Syndrome: A Single-Center Observational Study. Am J Respir Crit Care Med. 2020;201(10):1294-1297.

21. Henry BM, Lippi G. Poor survival with extracorporeal membrane oxygenation in acute respiratory distress syndrome (ARDS) due to coronavirus disease 2019 (COVID-19): Pooled analysis of early reports. J Crit Care. 2020;58:27-28.

22. Asada M, Yoshida M, Suzuki T, Hatachi Y, Sasaki T, Yasuda H, Nakayama K, et al. Macrolide antibiotics inhibit respiratory syncytial virus infection in human airway epithelial cells. Antiviral Res. 2009;83(2):191-200.

23. Retallack H, Di Lullo E, Arias C, Knopp KA, Laurie MT, Sandoval-Espinosa C, Mancia Leon WR, et al. Zika virus cell tropism in the developing human brain and inhibition by azithromycin. Proc Natl Acad Sci U S A. 2016;113(50):14408-14413.

24. Madrid PB, Panchal RG, Warren TK, Shurtleff AC, Endsley AN, Green CE, Kolokoltsov A, et al. Evaluation of Ebola virus inhibitors for drug repurposing. ACS Infect Dis. 2015;1(7):317-326.

25. Gautret P, Lagier JC, Parola P, Hoang VT, Meddeb L, Mailhe M, Doudier B, et al. Hydroxychloroquine and azithromycin as a treatment of COVID-19: results of an open-label non-randomized clinical trial. Int J Antimicrob Agents. 2020:105949. 
26. Simpson TF, Kovacs RJ, Steckler EC. Ventricular arrhythmia risk due to hydroxychloroquine-azithromycin treatment for COVID-19. Cardiology. 2020.

27. Magagnoli J, Narendran S, Pereira F, Cummings T, Hardin JW, Sutton SS, Ambati J. Outcomes of hydroxychloroquine usage in United States veterans hospitalized with Covid-19. medRxiv. 2020

28. Food and Drug Administration. https://www.fda.gov/media/137564/download.

29. Sanders JM, Monogue ML, Jodlowski TZ, Cutrell JB. Pharmacologic treatments for coronavirus disease 2019 (COVID-19): A Review. JAMA. 2020.

30. Tanaka T, Narazaki M, Kishimoto T. Immunotherapeutic implications of IL-6 blockade for cytokine storm. Immunotherapy. 2016;8(8):959-970.

31. Chu CM, Cheng VC, Hung IF, Wong MM, Chan KH, Chan KS, Kao RY, et al. Role of lopinavir/ritonavir in the treatment of SARS: initial virological and clinical findings. Thorax. 2004;59(3):252-256.

32. de Wilde AH, Jochmans D, Posthuma CC, ZevenhovenDobbe JC, van Nieuwkoop S, Bestebroer TM, van den Hoogen BG, et al. Screening of an FDA-approved compound library identifies four small-molecule inhibitors of Middle East respiratory syndrome coronavirus replication in cell culture. Antimicrob Agents Chemother. 2014;58(8):4875-4884.

33. Cao B, Wang Y, Wen D, Liu W, Wang J, Fan G, Ruan L, et al. A Trial of Lopinavir-Ritonavir in Adults Hospitalized with Severe Covid-19. N Engl J Med. 2020;382(19):1787-1799.

34. Hurst M, Faulds D. Lopinavir. Drugs. 2000;60(6):13801381.

35. Carlos D, Spiller F, Souto FO, Trevelin SC, Borges VF, de Freitas A, Alves-Filho JC, et al. Histamine h2 receptor signaling in the pathogenesis of sepsis: studies in a murine diabetes model. J Immunol. 2013;191(3):1373-1382.

36. Ciz M, Lojek A. Modulation of neutrophil oxidative burst via histamine receptors. Br J Pharmacol. 2013;170(1):1722.

37. Jafarzadeh A, Nemati M, Khorramdelazad H, Hassan ZM. Immunomodulatory properties of cimetidine: Its therapeutic potentials for treatment of immune-related diseases. Int Immunopharmacol. 2019;70:156-166.

38. Kapinska-Mrowiecka M, Turowski G. [Efficacy of cimetidine in treatment of Herpes zoster in the first 5 days from the moment of disease manifestation]. Pol Tyg Lek. 1996;51(23-26):338-339.

39. Kurzrock R, Auber M, Mavligit GM. Cimetidine therapy of herpes simplex virus infections in immunocompromised patients. Clin Exp Dermatol. 1987;12(5):326-331.

40. Harcourt JP, Worley G, Leighton SE. Cimetidine treatment for recurrent respiratory papillomatosis. Int J Pediatr Otorhinolaryngol. 1999;51(2):109-113.

41. Zhang W, Wang J, Su B, Li R, Ding Z, Kang Y, Wang B. Cimetidine augments Th1/Th2 dual polarized immune responses to recombinant HBV antigens. Vaccine. 2011;29(29-30):4862-4868.

42. Bourinbaiar AS, Fruhstorfer EC. The effect of histamine type 2 receptor antagonists on human immunodeficiency virus (HIV) replication: identification of a new class of antiviral agents. Life Sci. 1996;59(23):PL365-PL370.

43. Liu YJ. IPC: professional type 1 interferon-producing cells and plasmacytoid dendritic cell precursors. Annu Rev Immunol. 2005;23:275-306.

44. Chan JF, Yao Y, Yeung ML, Deng W, Bao L, Jia L, Li $\mathrm{F}$, et al. Treatment with lopinavir/ritonavir or interferonbetalb improves outcome of MERS-CoV infection in a nonhuman primate model of common marmoset. J Infect Dis. 2015;212(12):1904-1913.

45. Stockman LJ, Bellamy R, Garner P. SARS: systematic review of treatment effects. PLoS Med. 2006;3(9):e343.

46. Channappanavar R, Fehr AR, Zheng J, Wohlford-Lenane C, Abrahante JE, Mack M, Sompallae R, et al. IFN-I response timing relative to virus replication determines MERS coronavirus infection outcomes. J Clin Invest. 2019;130:3625-3639.

47. Luke TC, Kilbane EM, Jackson JL, Hoffman SL. Metaanalysis: convalescent blood products for Spanish influenza pneumonia: a future $\mathrm{H} 5 \mathrm{~N} 1$ treatment? Ann Intern Med. 2006;145(8):599-609.

48. Zhang J, Xie B, Hashimoto K. Current status of potential therapeutic candidates for the COVID-19 crisis. Brain Behav Immun. 2020.

49. Dodd RY. Emerging pathogens and their implications for the blood supply and transfusion transmitted infections. Br J Haematol. 2012;159(2):135-142.

50. Hung IF, To KK, Lee CK, Lee KL, Chan K, Yan WW, Liu $\mathrm{R}$, et al. Convalescent plasma treatment reduced mortality in patients with severe pandemic influenza A (H1N1) 2009 virus infection. Clin Infect Dis. 2011;52(4):447456.

51. Mair-Jenkins J, Saavedra-Campos M, Baillie JK, Cleary P, Khaw FM, Lim WS, Makki S, et al. The effectiveness of convalescent plasma and hyperimmune immunoglobulin for the treatment of severe acute respiratory infections of viral etiology: a systematic review and exploratory meta-analysis. J Infect Dis. 2015;211(1):80-90.

52. Roback JD, Guarner J. Convalescent plasma to treat COVID-19: possibilities and challenges. JAMA. 2020.

53. Bloch EM, Shoham S, Casadevall A, Sachais BS, Shaz B, Winters JL, van Buskirk C, et al. Deployment of convalescent plasma for the prevention and treatment of COVID-19. J Clin Invest. 2020.

54. Shen C, Wang Z, Zhao F, Yang Y, Li J, Yuan J, Wang F, et al. Treatment of 5 critically ill patients with COVID-19 with convalescent plasma. JAMA. 2020.

55. Duan K, Liu B, Li C, Zhang H, Yu T, Qu J, Zhou M, et al. Effectiveness of convalescent plasma therapy in severe COVID-19 patients. Proc Natl Acad Sci U S A. 2020;117(17):9490-9496.

56. Cheng Y, Wong R, Soo YO, Wong WS, Lee CK, Ng MH, Chan $\mathrm{P}$, et al. Use of convalescent plasma therapy in SARS patients in Hong Kong. Eur J Clin Microbiol Infect Dis. 2005;24(1):44-46.

57. Chen L, Xiong J, Bao L, Shi Y. Convalescent plasma as a potential therapy for COVID-19. Lancet Infect Dis. 2020;20(4):398-400.

58. Casadevall A, Pirofski LA. Antibody-mediated regula- 
tion of cellular immunity and the inflammatory response. Trends Immunol. 2003;24(9):474-478.

59. Xu X, Han M, Li T, Sun W, Wang D, Fu B, Zhou Y, et al. Effective treatment of severe COVID-19 patients with tocilizumab. Proc Natl Acad Sci U S A. 2020;117(20):1097010975.

60. Chang JC. Sepsis and septic shock: endothelial molecular pathogenesis associated with vascular microthrombotic disease. Thromb J. 2019;17:10.

61. Hou PC, Filbin MR, Wang H, Ngo L, Huang DT, Aird WC, Yealy DM, et al. Endothelial permeability and hemostasis in septic shock: results from the ProCESS trial. Chest. 2017;152(1):22-31.

62. Johansson PI, Stensballe J, Ostrowski SR. Shock induced endotheliopathy (SHINE) in acute critical illness - a unifying pathophysiologic mechanism. Crit Care. 2017;21(1):25.

63. Nguyen TC, Carcillo JA. Bench-to-bedside review: thrombocytopenia-associated multiple organ failure - a newly appreciated syndrome in the critically ill. Crit Care. 2006;10(6):235.

64. Busund R, Koukline V, Utrobin U, Nedashkovsky E. Plasmapheresis in severe sepsis and septic shock: a prospective, randomised, controlled trial. Intensive Care Med. 2002;28(10):1434-1439.

65. Monard C, Rimmele T, Ronco C. Extracorporeal blood purification therapies for sepsis. Blood Purif. 2019;47(Suppl 3):1-14.

66. Ma J, Xia P, Zhou Y, Liu Z, Zhou X, Wang J, Li T, et al. Potential effect of blood purification therapy in reducing cytokine storm as a late complication of critically ill COVID-19. Clin Immunol. 2020;214:108408.

67. Blanco JL, Ambrosioni J, Garcia F, Martinez E, Soriano A, Mallolas J, Miro JM, et al. COVID-19 in patients with HIV: clinical case series. Lancet HIV. 2020;7(5):e314e316.

68. Ford N, Vitoria M, Rangaraj A, Norris SL, Calmy A, Doherty M. Systematic review of the efficacy and safety of antiretroviral drugs against SARS, MERS or COVID-19: initial assessment. J Int AIDS Soc. 2020;23(4):e25489.

69. Au K, Reed G, Curtis JR, Kremer JM, Greenberg JD, Strand V, Furst DE, et al. High disease activity is associated with an increased risk of infection in patients with rheumatoid arthritis. Ann Rheum Dis. 2011;70(5):785791.

70. Monti S, Balduzzi S, Delvino P, Bellis E, Quadrelli VS, Montecucco C. Clinical course of COVID-19 in a series of patients with chronic arthritis treated with immunosuppressive targeted therapies. Ann Rheum Dis. 2020;79(5):667-668.

71. Venerito V, Lopalco G, Iannone F. COVID-19, rheumatic diseases and immunosuppressive drugs: an appeal for medication adherence. Rheumatol Int. 2020;40(5):827-828.

72. Li F, Cai J, Dong N. First cases of COVID-19 in heart transplantation from China. J Heart Lung Transplant. 2020;39(5):496-497.

73. Romanelli A, Mascolo S. Immunosuppression drug-related and clinical manifestation of Coronavirus disease 2019: A therapeutical hypothesis. Am J Transplant. 2020.
74. Fernandez-Ruiz M, Andres A, Loinaz C, Delgado JF, Lopez-Medrano F, San Juan R, Gonzalez E, et al. COVID-19 in solid organ transplant recipients: A single-center case series from Spain. Am J Transplant. 2020.

75. Akalin E, Azzi Y, Bartash R, Seethamraju H, Parides M, Hemmige V, Ross M, et al. Covid-19 and kidney transplantation. N Engl J Med. 2020.

76. FIGO (International Federation of Gynecology and Obstetrics). https:/www.figo.org/news/figo-covid-19-resourceS.

77. Poon LC, Yang H, Kapur A, Melamed N, Dao B, Divakar H, McIntyre HD, et al. Global interim guidance on coronavirus disease 2019 (COVID-19) during pregnancy and puerperium from FIGO and allied partners: Information for healthcare professionals. Int J Gynaecol Obstet. 2020;149(3):273-286.

78. Chen H, Guo J, Wang C, Luo F, Yu X, Zhang W, Li J, et al. Clinical characteristics and intrauterine vertical transmission potential of COVID-19 infection in nine pregnant women: a retrospective review of medical records. Lancet. 2020;395(10226):809-815.

79. Zhu H, Wang L, Fang C, Peng S, Zhang L, Chang G, $\mathrm{Xia} \mathrm{S}$, et al. Clinical analysis of 10 neonates born to mothers with 2019-nCoV pneumonia. Transl Pediatr. 2020;9(1):51-60.

80. Basile C, Combe C, Pizzarelli F, Covic A, Davenport A, Kanbay M, Kirmizis D, et al. Recommendations for the prevention, mitigation and containment of the emerging SARS-CoV-2 (COVID-19) pandemic in haemodialysis centres. Nephrol Dial Transplant. 2020;35(5):737-741.

81. Adapa S, Aeddula NR, Konala VM, Chenna A, Naramala S, Madhira BR, Gayam V, Balla M, Muppidi V, Bose S. COVID-19 associated renal failure and challenges to deliver optimal renal replacement therapy in hospital and outpatient settings: a review of literature. J Clin Med Res. 2020. [Article in Press].

82. Bose S, Adapa S, Konala VM, Gopal RH, Sohail S, Naramala S, Kondakindi H, et al. Atypical presentation of novel coronavirus disease 19 in a peritoneal dialysis patient. J Investig Med High Impact Case Rep. [Article in Press].

83. Ma Y, Diao B, Lv X, et al. 2019 Novel coronavirus disease in hemodialysis (HD) patients: report from one HD Center in Wuhan, China. https://www.medrxiv.org/conte $\mathrm{nt} / 10.1101 / 2020.02 .24 .20027201 \mathrm{v} 2$.

84. NIH U.S. National Library of Medicine. https://clinicaltrials.gov/ct2/results? cond=COVID\&Search=Apply\&r ecrs $=$ a\&age_v $=\&$ gndr $=\&$ type $=$ Intr\&rslt $=$. Accessed on April 28, $20 \overline{2} 0$.

85. https://www.medicalnewstoday.com/articles/covid19-new-drug-candidates-treatments-offer-reasons-forhope. Accessed on May 17, 2020.

86. https://www.forbes.com/sites/joshuacohen/2020/05/11/ pricing-of-covid-19-treatments-and-coronavirusvaccines/\#6c6f46f62865. Accessed May 17, 2020.

87. Hill A, Wang J, Levi J, Heath K, Fortunak J. Minimum costs to manufacture new treatments for COVID-19. J Virus Erad. 2020;6(2):61-69.

88. World Health Organization. https://www.who.int/docs/ 
default-source/coronaviruse/covid-strategy-update14april2020.pdf?sfvrsn=29da3ba0_6.

89. National coronavirus response. https://www.aei.org/wpcontent/uploads/2020/03/National-Coronavirus-Re-
sponse-a-Road-Map-to-Recovering-2.pdf.

90. Center for American Progress. https://www.americanprogress.org/issues/healthcare/news/2020/04/03/482613/ national-state-plan-end-coronavirus-crisis/. 